
\title{
25 Research Square \\ Chronic Social Stress Impairs Prefrontal Cortical Myelination in Juvenile Mice
}

\section{Xuan Sun}

Nanchang University

Qiu-Hui-Hong Yu

School of Life Sciences, Nanchang University

Fei Luo ( $\sim$ luofei@ncu.edu.cn )

Nanchang University

\section{Bao-Ming Li}

Institute of Brian Science and Department of psychology, School of Education, Hangzhou Normal University

\section{Research}

Keywords: Chronic social stress, Prefrontal cortex, Myelination, Juvenile mice

Posted Date: August 28th, 2020

DOI: https://doi.org/10.21203/rs.3.rs-54172/v1

License: (1) (1) This work is licensed under a Creative Commons Attribution 4.0 International License. Read Full License 


\section{Abstract}

The prefrontal cortex (PFC) is a key brain region mediating many cognitive functions, and its structures and functions are particularly vulnerable to stress. Chronic social defeat stress (CSD) has been proposed as a model of anxiety/depressive-like behaviors. In the present study, we examined whether CSD affects the myelination in the medial PFC (MPFC) in mice. Our results show that CSD dramatically reduced the myelin basic protein (MBP) staining in the MPFC, with no effect on the MBP-labeling in the motor cortex, striatum, hippocampus and corpus callosum. Consistently, the CSD mice demonstrated a significant increase in oligodendrocyte precursor cells ( $\mathrm{PDGFRa}^{+}$cells) and a significant decrease in mature oligodendrocytes ( $\mathrm{CC}^{+} / \mathrm{Olig}^{+}$cells) in the mPFC. The present study demonstrates that CSD impairs the differentiation of oligodendrocytes and myelination in the MPFC, suggesting a potential mechanism for stress-induced change in PFC-dependent behaviors.

\section{Introduction}

\section{HIGHLIGHTS}

- Chronic social defeat induces a severe deficit in the myelination of MPFC.

- Chronic social defeat impairs the OPC-to-OL differentiation in the MPFC.

- Chronic social defeat does not affect the myelination of other brain regions.

It is well established that social defeat induces anxiety [1, 2] and depressive-like behaviors [3]. Consistently, social defeat results in deficits in cognitive functions. For example, plundering-induced social pressure produces a severe deficit in object recognition learning [4]. Mice with social defeat exhibit a poor learning and memory abilities in T-maze and water maze tests [5].

Myelin provides trophic support for axons, maintains axonal integrity and enables rapid transmission of action potentials via saltatory conduction $[6,7]$. Axonal myelination in the prefrontal cortex (PFC) is the last step in ontogenetic development in vertebrate central nervous system [8], opening an opportunity windows for nurture experience to shape PFC functions. Abnormal myelination is linked to many neurological and psychiatric disorders [9].

The PFC is an associative cortex controlling high-level executive and cognitive functions [10]. A previous study in our laboratory reveals that neonatal maternal separation (NMS) stress causes a severe deficit in the myelination in the medial PFC (mPFC) of rats, and such deficit is mediated via Wnt signaling [11]. To know whether stress has a general impact on PFC myelination, the present study used a chronic social defeat (CSD) model to see if such stress also affects the myelination of the mPFC.

\section{Experimental Procedure}

\subsection{Ethics statement for the experiments}


All experiments were performed in strict accordance with the principles of laboratory animal care and use approved by the Nanchang University Animal Care and Use Committee guidelines. Mice were deeply anesthetized with pentobarbital sodium before sacrifice. All efforts were made to minimize the number of mice and minimize suffering.

\subsection{Animals}

All C57BL/ 6 mice were bred in our laboratory and retired breeder CD-1 mice (male, aged 8-9 month) were purchased from Beijing Vital River Laboratory Animal Technology Co., Ltd. Mice were housed under a temperature-controlled environment about $25^{\circ} \mathrm{C}$ and 12-hourlight/dark circles (lights on between 7:0019:00) with ad libitum access to food and water.

\subsection{Chronic social defeat (CSD)stress model}

The procedure of CSD included two phases: the phase to select aggressive male CD-1 mice and the phase to perform chronic social defeat stress. New purchased CD-1 mice were kept alone for 7 days without disturbing to acclimate new circumstances. Mice aged 3-4 weeks were used to select aggressive CD-1 mice. Three different mice were placed into the CD-1 mice home cage individually, and were allowed to contact with the host for 3 minutes. In the three sessions, CD-1 mice attacked C57BL/6J within 60 seconds in at least two consecutive sessions were used to perform the following experiment. Experimental C57BL/ 6 mouse was placed into the selected CD-1 home cage to allow the aggressive behaviors occurred. One minute later, the two mice were immediately separated by a perforated transparent acrylic partition to allow visual, olfactory and auditory contact for the remainder of 24 hours. C57BL/ 6 mice were introduced to unfamiliar aggressive CD-1 mice for consecutive 10 days.

\subsection{Immunofluorescence}

Experimental C57BL/ 6 mice were deeply anesthetized by $1 \%$ pentobarbital sodium ( $50 \mathrm{mg} / \mathrm{kg}$, i.p.) and transcardially perfused with $0.9 \%$ sodium chloride solution followed by $4 \%$ paraformaldehyde solution (4\% PFA, pH = 7.35). Brains were dissected out, post-fixed in 4\% PFA and gradient dehydrated in $10 \%, 20 \%$, $30 \%(\mathrm{w} / \mathrm{v})$ sucrose solution and were then sliced into $30 \mu \mathrm{m}$ coronal sections with the freezing microtome (Leica CM900, Germany). The sections were blocked in blocking solution containing $10 \%$ bovine serum albumin and then incubated with primary antibody at $4{ }^{\circ} \mathrm{C}$ overnight. After rinse for three times for at least 30 minutes with $0.01 \mathrm{M}$ PBS, the slices were incubated with secondary antibody for 2 hours at room temperature. After three rinses, slices were incubated with DAPI solution for 5 minutes and then followed by washout. The sections were cover-slipped with fluorescent mounting medium (Sigma-Aldrich, F4680) and imaged with confocal laser microscope (Olympus FV10-ASW, Japan).

Following antibodies were used: rabbit anti-MBP (1:1000, Abcam, ab40390), rabbit anti-Olig2 (1:1000, Millipore, AB9610), rat anti-PDGFRa (1:500, BD Bioscience, 558774), mouse anti-CC1 (1:100, Millipore, OP80), donkey anti-mouse AlexaFlour ${ }^{\mathrm{TM}} 488$ (1:500, Thermo Fisher, A21206), donkey anti-rabbit AlexaFlour $^{\text {TM }} 568$ (1:500, Thermo Fisher, A10042), donkey anti-rat AlexaFlour ${ }^{\text {TM }} 488$ (1:500, Thermo Fisher, A21208). 


\subsection{Western blotting analysis}

After deeply anesthetized by $1 \%$ pentobarbital sodium $(50 \mathrm{mg} / \mathrm{kg}$, i.p.), mice were rapidly decapitated and the brains were dissected out. After washed by phosphate-buffered saline (PBS), the tissues from mPFC were ground into homogenate in RIPA lysate with phenylmethanesulfonyl fluoride (PMSF, Sigma). After centrifugation at $12,000 \mathrm{rpm}$ for 10 minutes at $4^{\circ} \mathrm{C}$, the concentration of supernatant protein was measured using the BCA Protein Kit (Thermo Scientific) according to the manufacturer's instructions. Equivalent of total proteins was loaded onto $12 \%$ SDS-PAGE to separation and then transferred onto polyvinylidene difluoride (PVDF) membrane (Merck Millipore). Membrane was blocked with $5 \%$ non-fat milk at room temperature for 2 hours. Primary antibodies (rabbit anti-MBP, 1:500, Abcam, ab40390; mouse anti-GAPDH, 1:1000, Bio-rad, MCA4739) were incubated at $4^{\circ} \mathrm{C}$ overnight, then washed three times in TBST at least 15 minutes and incubated with secondary antibodies (Goat anti-mouse IgG HRP, 1:3000, CWBiotech, Cw0102s; Goat anti-rabbit IgG HRP, 1:3000, CWBiotech, Cw0103s) for one hour at room temperature. Signals were visualized using the Gel Doc ${ }^{\text {TM }}$ EZ System (Bio-Rad) and Image $J$ was used to analyze the relative protein concentration.

\subsection{Statistical Analysis}

Statistical analysis was performed using GraphPad Prism 6. Unpaired Student's t test was applied. $P<$ 0.05 was considered to be statistically significant.

\section{Results}

\subsection{Myelination rapidly increases during P10-30 in the MPFC}

We first examined the process of myelination in the mouse mPFC. Immunohistochemistry for myelin basic protein (MBP) showed that few myelination could be seen in the MPFC at P10, and the myelination increased from P10 through P30, and reached a relatively stable level during P40-P60 (Fig. 1B). Because myelin is formed by oligodendrocytes (OLs) in the central nervous system, we next analyzed the change in OLs in the mPFC. We found that the number of OLs, as indicated by Olig2 staining, significantly increased during P10-P30 (Fig. 1C and Fig. 1D, P10: $177.2 \pm 9.6, n=6 ; P 20: 313.2 \pm 8.8, n=6, P<0.01$ vs P10; P30: $474.7 \pm 13.9, n=6, P<0.01$ vs $P 20)$. Thus, postnatal day $10-30$ is a critical period for the mouse $\mathrm{MPFC}$ to be myelinated.

\subsection{Chronic social defeat stress impairs myelination in the MPFC}

We then examined the effect of chronic social defeat (CSD) stress on the myelination status in mPFC at P30, using MBP immunohistochemical staining. As shown in Fig. 2, CSD significantly reduced the myelin level in the prelimbic cortex (PL) (Fig. 2A, Control (CON): $29.4 \pm 1.0 \%, n=6$; CSD: $21.2 \pm 1.2 \%, n=6 ; P<$ 0.01 vs CON) and the infralimbic cortex (IL) of the mPFC (Fig. 2B, CON: $18.3 \pm 0.9 \%, n=6$; CSD: $13.7 \pm$ $1.6 \%, n=6 ; P<0.05$ vs $C O N)$. Consistently, western blot analysis showed that MBP level was significantly reduced in the mPFC of CSD mice (Fig. 2 C, CON: $1.1 \pm 0.2, n=3$; CSD: $0.4 \pm 0.1, n=3 ; P<0.05$ vs CON). 
We next investigated the effect of CSD on the myelination of other brain structures at P30. We found that CSD mice demonstrated comparable myelination level (MBP-staining) in the motor cortex (Fig. 3A and $3 B, C O N: 38.3 \pm 0.6, n=6$; CSD: $38.2 \pm 0.6, n=6 ; P>0.05$ vs CON), comparable thickness of the corpus callosum (Fig. 3 C and 3D, CON: $286.7 \pm 28.2 \mu \mathrm{m}, \mathrm{n}=6$; CSD: $275.0 \pm 25.9 \mu \mathrm{m}, \mathrm{n}=6$; $\mathrm{P}>0.05$ vs CON), comparable myelination level in the striatum (Fig. 3E and 3F, CON: $20.1 \pm 0.5 \%, n=6$; CSD: $20.3 \pm 0.6 \%, n$ $=6 ; \mathrm{P}>0.05$ vs CON) and in the hippocampus (Fig. $3 \mathrm{G}$ and $3 \mathrm{H}, \mathrm{CON}: 15.0 \pm 1.3, \mathrm{n}=6$; CSD: $15.8 \pm 1.0, \mathrm{n}=$ $6 ; P>0.05$ vs $C O N$ ), indicating that CSD produces no impact on the myelination of these brain structures.

\subsection{CSD increasesOPLs and reduces mature OLs in the MPFC}

Oligodendrocyte precursor cells (OPCs) arise from neuroepithelial progenitors in the ventral neural tube, and they migrate, proliferate and ultimately differentiate to form mature OLs [12,13]. We then addressed whether CSD affects the number of OPCs and OLs in the MPFC. As shown in Fig. 4A, the CSD mice exhibited a significant increase in OPCs, as indicated by PDGFRa ${ }^{+}$, in the prelimbic (PL) region (Fig. 4A, CON: $102.0 \pm 5.6, n=6 ; C S D, 147.5 \pm 8.6, n=6 ; P<0.01$ vs $C O N$ ) and the infralimbic (IL) region of the mPFC (Fig. 4A, CON: $86.0 \pm 3.6, n=6$; CSD: $101.3 \pm 3.7, n=6 ; P<0.05$ vs CON). Conversely, the CSD mice showed a significant decrease in mature OLs, as indicated by $O$ lig2 ${ }^{+} \mathrm{CC}^{+}$, in the PL region (Fig. $4 \mathrm{~B}, \mathrm{CON}$ : $30.0 \pm 1.5, n=6$; CSD: $22.8 \pm 1.5, n=6, P<0.01$ vs CON) and the IL region (Fig. $4 B, C O N: 17.8 \pm 1.7, n=6$; CSD: $12.2 \pm 1.2, n=6 ; P<0.05$ vs $C O N)$. This result suggests that CSD impairs the OPC-to-OL differentiation in the mPFC.

\section{Discussion}

Axonal myelination is important for normal functions of the central neural system [14, 15]. Myelination plasticity could occur in response to early-life and social experience $[16,17]$. In humans, the myelination in the brain continues from childhood through adulthood, extending until at least age 30 [16]. Manabu et al. reported that juvenile social experience influences the maturation of OLs and the myelination of mPFC in mice during a "critical period" between P21 and P35 [17]. The present study revealed that the mPFC myelination of mice occurs during P10-P30 and reaches a stable level during P40-P60, consistent with the temporal pattern of myelination seen in the MPFC of rats [11].

Considering that the myelination of the mPFC increases rapidly at P10-P30, we then examined the effect of CSD on the mPFC myelination at this critical stage. Indeed, the myelin level of the MPFC in CSD mice was significantly lower than in controls. A similar result has been reported in the mice subjected to CSD [18]. However, the present study shows that CSD did not affect the myelination in the motor cortex, corpus callosum, striatum and hippocampus. This phenomenon again indicates that the prefrontal cortex is quite fragile with early-life stress in term of myelination, suggesting that early-life social experience has a huge ability to shape the structures, and therefore, the functions of the PFC. 
As myelinating cells in the central nervous system, mature OLs carry out myelination process [7]. OPCs divide and differentiate into OLs in postnatal brain [19]. The present study found that CSD stress caused a significant increase in the number of OPCs, and a significant decrease in the number of mature OLs. It is possible that the signaling mechanism of the OPC-to-OL differentiation was affected somehow by the CSD stress. Indeed, the previous study by Yang et al. demonstrated that Wnt signaling pathway involves in the neonatal maternal separation induced suppression of OPC-to-OL differentiation in the MPFC of rats [11]. It remains to be confirmed whether such mechanism also applies for the CSD-induced deficit of OPCto-OL differentiation.

It has been revealed that dysregulation of the hypothalamic-pituitary-adrenal (HPA) axis is involved in stress-related declines in mood and cognitive functions [20,21]. Several laboratories have reported that adrenal glucocorticoids could directly target OLs, resulting in OL-related pathology $[22,23]$. Considering that CSD stress produces a prolonged elevation in the level of glucocorticoids [24], an abnormal change in the activity of the HPA axis might account for the altered function of OLs in MPFC myelination.

In summary, chronic social defeat stress reduces the production of mature OLs in the MPFC of mice and impairs the myelination of the mPFC, suggesting that a chronic stress, whether it is a neonatal maternal separation stress or a chronic social defeat one, selectively destroys prefrontal cortical myelination. Such destructive effect might be one of underlying mechanisms for stress-induced psychiatric disorders seen in humans.

\section{Abbreviations}

CSD, chronic social defeat stress; MBP, myelin basic protein; PDGFRa, platelet derived growth factor receptor a; PL, prelimbic cortex; IL, infralimbic cortex.

\section{Declarations}

\section{Acknowledgements}

The authors thank Zong-Yue Cheng, You-Jun Yang and Jie Luo for experimental assistance and discussion.

\section{Author contributions}

Bao-Ming Li and Fei Luo designed the experiments. Xuan Sun and Qiu-Hui-Hong Yu performed the experiments. Xuan Sun and Fei Luo drafted the manuscript. Bao-Ming Li revised the manuscript. All authors approved the final version of the manuscript.

\section{Funding}

This study was supported by the National Natural Science Foundation of China (31971035, 31771182, 81471116 and 81560196) and the Natural Science Foundation of Jiangxi Province (20171ACB20002). 
Availability of data and materials

The data generated or analyzed are included in this published article.

\section{Ethics approval}

All experiments were approved by the Nanchang Animal Care and Use Committee and conducted according to the Guidelines for the Care and Use of Laboratory Animals.

\section{Consent for publication}

Not applicable.

\section{Competing interests}

The authors declare that they have no competing interests.

\section{References}

1. Kinsey SG, Bailey MT, Sheridan JF, Padgett DA, Avitsur R. Repeated social defeat causes increased anxiety-like behavior and alters splenocyte function in C57BL/6 and CD-1 mice. Brain, behavior, and immunity. 2007;21:458-66.

2. Ashley D, David T, Keith W, Amanda C, Jonathan C, Jason G, et al. The effects of chronic social defeat stress on mouse self-grooming behavior and its patterning. Behavioural Brain Research. 2010;208:553-9.

3. Schloesser RJ, Lehmann M, Martinowich K, Manji HK, Herkenham M. Environmental enrichment requires adult neurogenesis to facilitate the recovery from psychosocial stress. Molecular Psychiatry. 2010;15:1152.

4. Wissam EH, Sylvie P, Guy G, Catherine B. Impaired memory following predatory stress in mice is improved by fluoxetine. Progress in Neuropsychopharmacology \& Biological Psychiatry. 2004;28:123-8.

5. Yu T, Guo M, Garza J, Rendon S, Sun XL, Zhang W, et al. Cognitive and neural correlates of depression-like behaviour in socially defeated mice: an animal model of depression with cognitive dysfunction. Int. J. Neuropsychopharmacol. 2011;14:303-17.

6. Bradl M, Lassmann H. Oligodendrocytes: biology and pathology. Acta Neuropathologica. 2010;119:37-53.

7. Nave KA, Werner HB. Myelination of the nervous system: mechanisms and functions. Annu Rev Cell Dev Biol. 2014;30:503-33.

8. Klaus-Armin N. Myelination and support of axonal integrity by glia. Nature. 2010;468:244.

9. Baumann N, Pham-Dinh D. Biology of oligodendrocyte and myelin in the mammalian central nervous system. Physiological reviews. 2001:81:871-927. 
10. Miller EK. The prefrontal cortex: complex neural properties for complex behavior. Neuron. 1999;22:1517.

11. Yang $Y$, Cheng Z, Tang $H$, Jiao $H$, Sun $X$, Cui Q, et al. Neonatal maternal separation impairs prefrontal cortical myelination and cognitive functions in rats through activation of Wnt signaling. Cereb Cortex. 2017;27:2871.

12. Dimou L, Gallo V. NG2-glia and their functions in the central nervous system. Glia. 2015;63:1429-51.

13. Hill RA, Akiko N. NG2 cells (polydendrocytes): listeners to the neural network with diverse properties. Glia. 2014;62:1195-1210.

14. Weickenmeiera J, Rooij Rd, Budday S, Ovaert TC, Kuhl E. The mechanical importance of myelination in the central nervous system. Journal of the mechanical behavior of biomedical materials. 2017;76:119-24.

15. Nickel M, Gu C. Regulation of Central Nervous System Myelination in Higher Brain Functions. Neural Plasticity. 2018 2018:1-12.

16. R Douglas F. Myelination: an overlooked mechanism of synaptic plasticity? Neuroscientist. 2005;11:528-31.

17. Manabu M, Rosen KM, Susumu I, Gabriel C. A critical period for social experience-dependent oligodendrocyte maturation and myelination. Science. 2012;337:1357-60.

18. Lehmann ML, Weigel TK, Elkahloun AG, Herkenham M. Chronic social defeat reduces myelination in the mouse medial prefrontal cortex. Scientific Reports. 2017;7:46548.

19. Zhu X, Hill AR, Dirk D, Mila K, Ryusuke S, Akiko N. Age-dependent fate and lineage restriction of single NG2 cells. Development. 2011;138:745-53.

20. Holsboer F, von Bardeleben U, Wiedemann K, Muller OA, Stalla GK. Serial assessment of corticotropin-releasing hormone response after dexamethasone in depression. Implications for pathophysiology of DST nonsuppression. Biol Psychiatry. 1987;22:228-34.

21. McEwen BS, Stellar E. Stress and the individual. Mechanisms leading to disease. Arch Intern Med. 1993;153:2093-101.

22. Alonso G. (2000) Prolonged corticosterone treatment of adult rats inhibits the proliferation of oligodendrocyte progenitors present throughout white and gray matter regions of the brain. Glia. 2000;31:219-31.

23. Miyata S, Taniguchi M, Koyama Y, Shimizu S, Tanaka T, Yasuno F, et al. Association between chronic stress-induced structural abnormalities in Ranvier nodes and reduced oligodendrocyte activity in major depression. Sci Rep. 2016;6:23084.

24. Lehmann ML, Brachman RA, Martinowich K, Schloesser RJ, Herkenham M. Glucocorticoids orchestrate divergent effects on mood through adult neurogenesis. J Neurosci. 2013;33:2961-72.

\section{Figures}


A

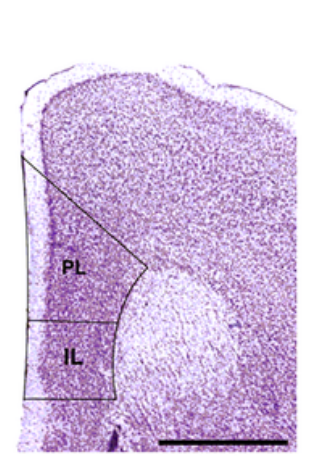

B
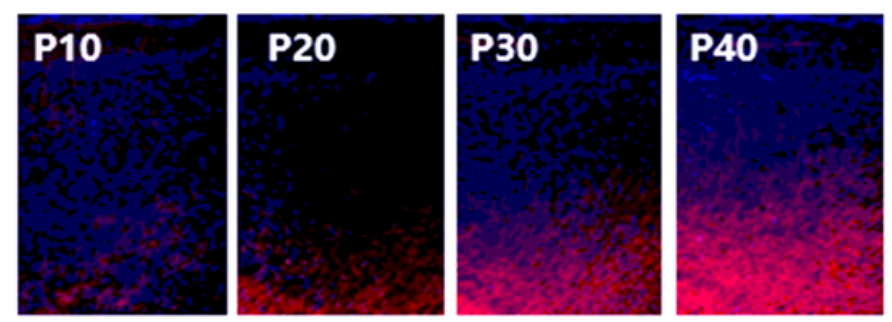

P60

Superficial

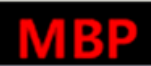

DAPI

Deep

C

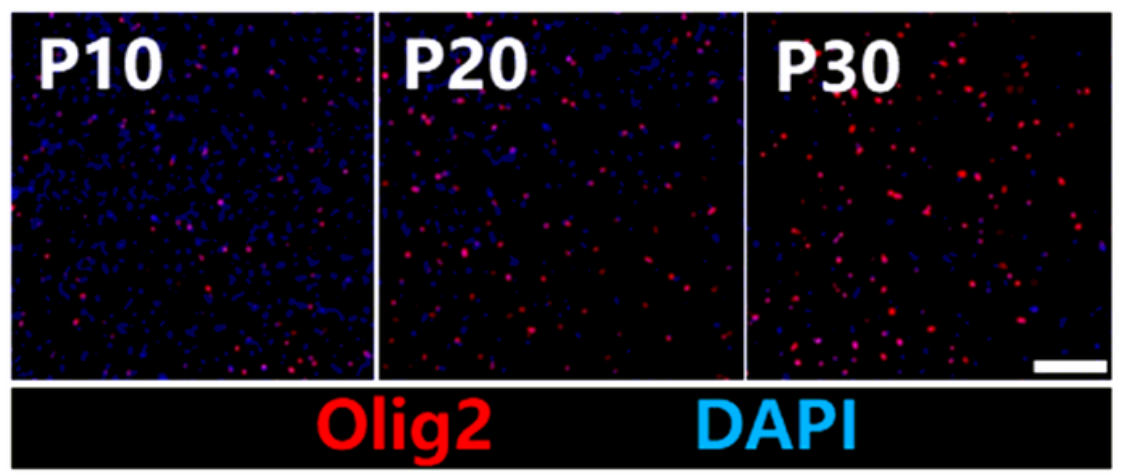

D

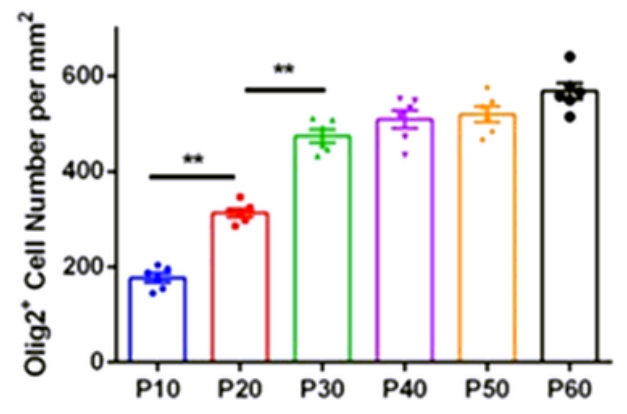

Figure 1

Myelination and oligodendrocytes increase at P10-30 in the mouse mPFC (A) A brain section showing the location of the medial prefrontal cortex (mPFC) of mouse. PL, prelimbic cortex; IL, infralimbic cortex. (B) Immunohistochemical staining of myelin basic protein (MBP) in the MPFC at different postnatal days. (C) Immunostaining of Olig2+ cells in the MPFC at different postnatal days. (D) Histogram showing the numbers of Olig2+ cells in the mPFC at different postnatal days. Scale bar: $200 \mu \mathrm{m}$. Data are shown as mean \pm SEM. ${ }^{* *} \mathrm{P}<0.01$. 

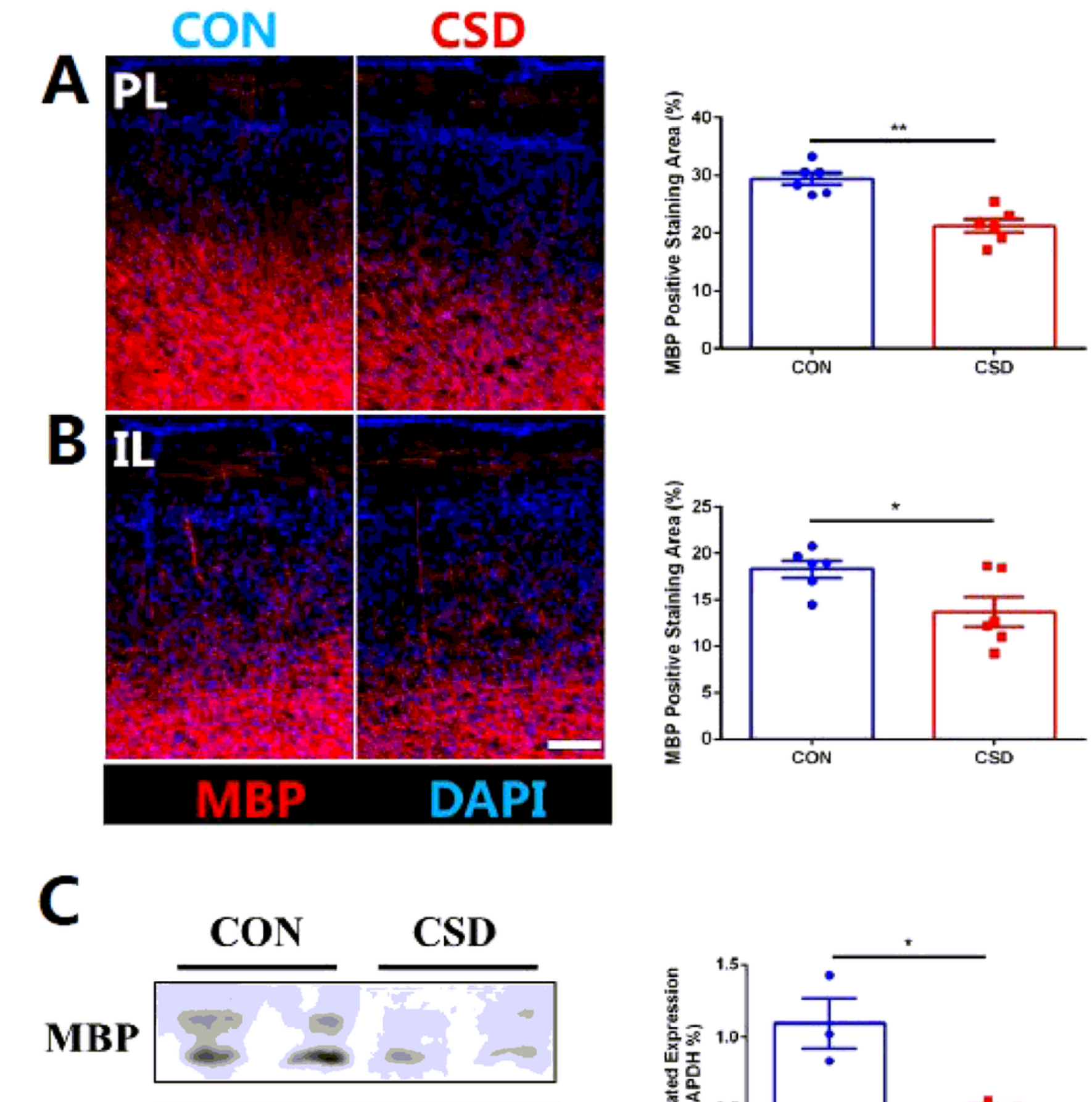

GAPDH

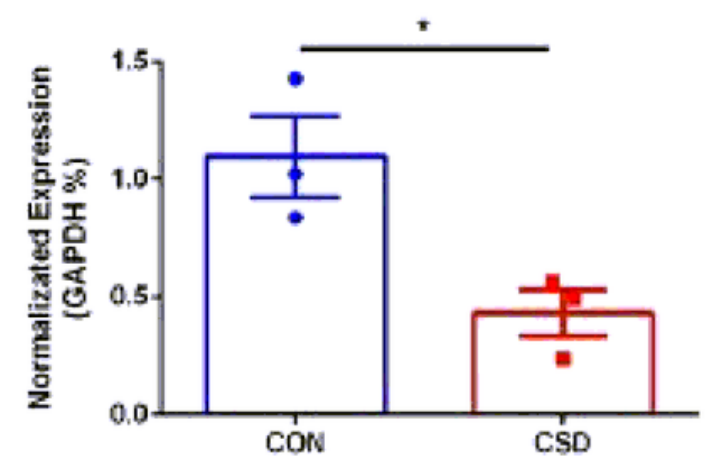

Figure 2

CSD stress impairs the myelination in the mouse mPFC (A) Immunohistochemical staining (left) and positive staining area of MBP (right) in the prelimbic cortex (PL) at postnatal day 30. (B) Immunohistochemical staining of MBP (left) and the positive staining area of MBP (right) in the infralimbic cortex (IL) at postnatal day 30. (C) Western blotting (left) and quantification analysis (right) 
for MBP in the PL and IL at postnatal day 30. CON: Control; CSD: Chronic social defeat stress. Scale bar: $150 \mu \mathrm{m}$. Data are shown as mean \pm SEM. ${ }^{*} \mathrm{P}<0.05 ;{ }^{*} \mathrm{P}<0.01$.
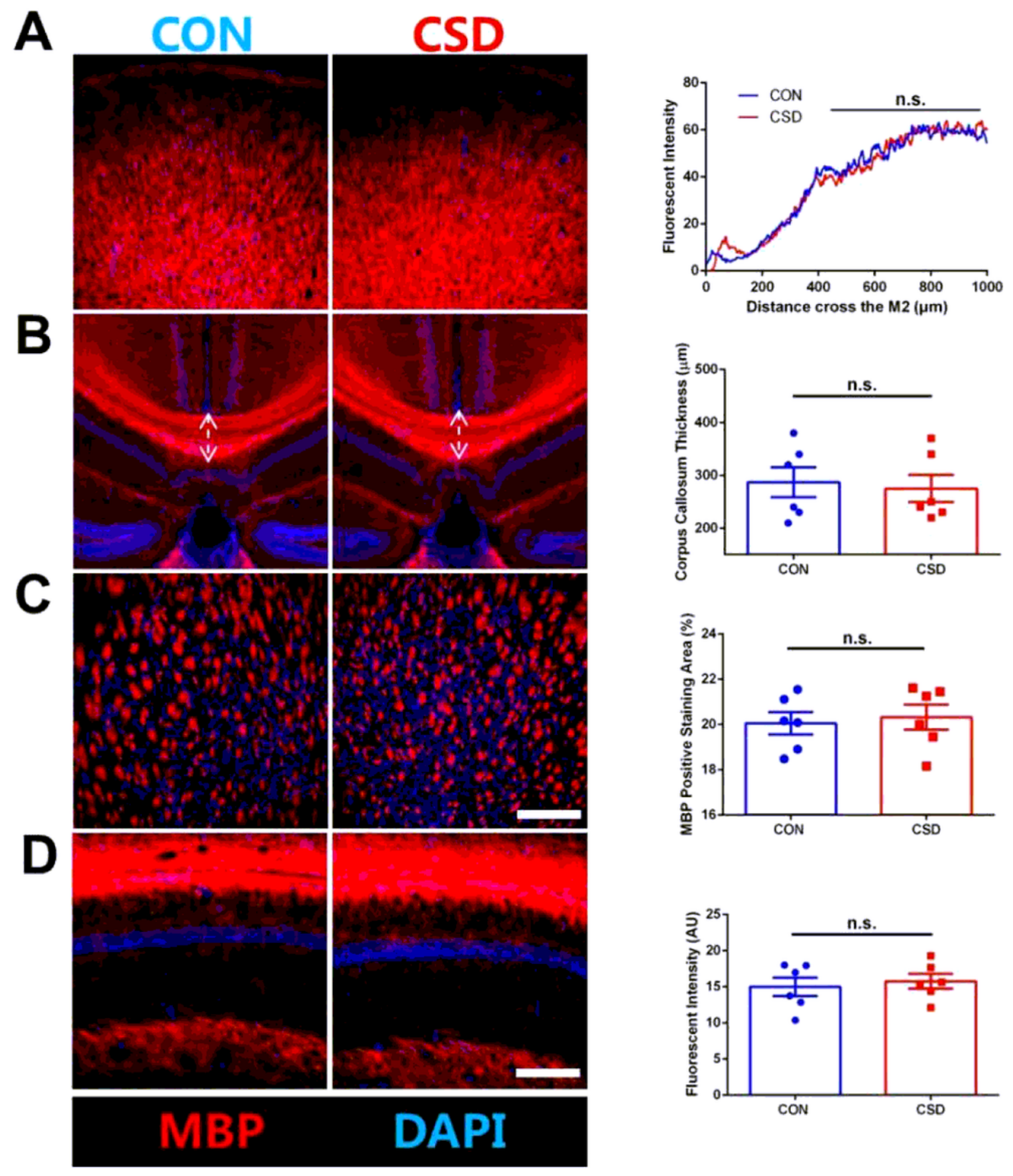

Figure 3

CSD stress has no impact on the myelination in the motor cortex, corpus callosum, striatum and hippocampus (A) Confocal image (left) and fluorescence intensity of MBP (right) in the motor cortex at postnatal day 30. No difference was seen between CSD and Control mice. (B) Confocal image of MBP in 
the corpus callosum (left) and thickness of the corpus callosum (right) at postnatal day 30 . No difference was seen between CSD and Control mice. (C) Confocal image (left) and staining area of MBP (right) in the striatum at postnatal day 30. No difference was seen between CSD and Control mice. (D) Confocal image (left) and fluorescence intensity of MBP (right) in the hippocampus at postnatal day 30 . No difference was seen between CSD and Control mice. CON: Control; CSD: Chronic social defeat stress; Scale bar: $200 \mu \mathrm{m}$ in Figure 3A-3C and $100 \mu \mathrm{m}$ in Figure 3D. Data are shown as mean \pm SEM. ns: no significance.

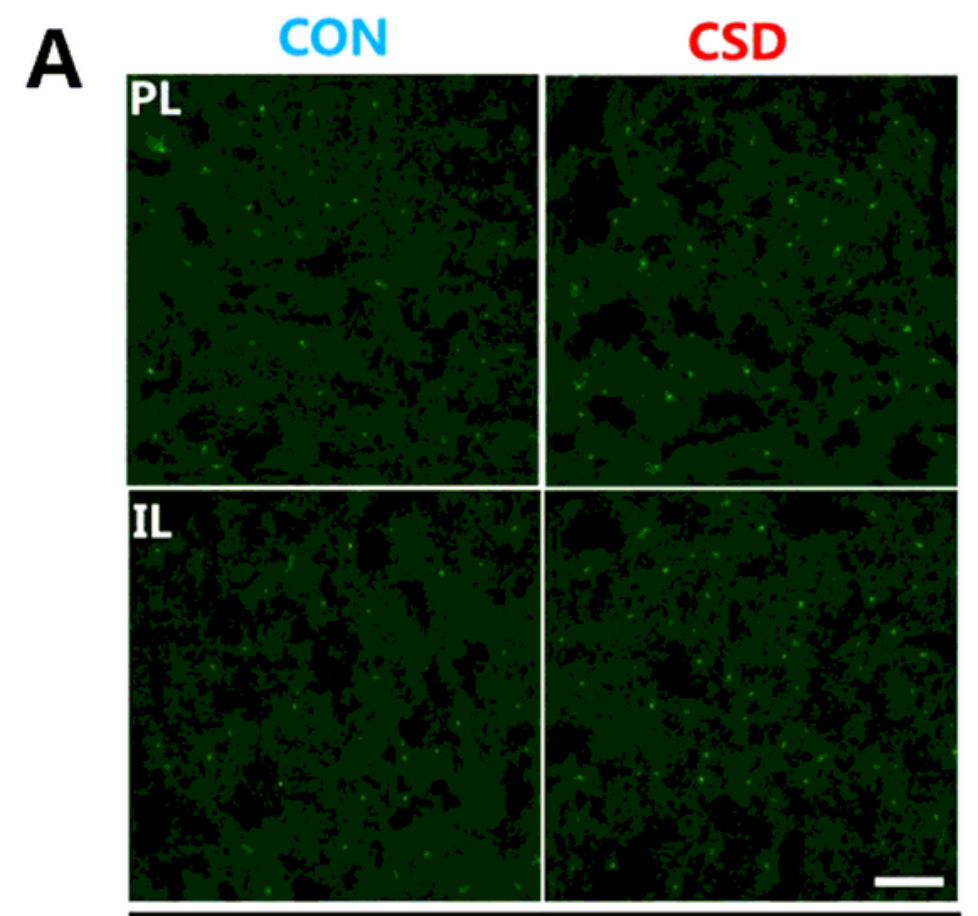

\section{PDGFR $\alpha$}
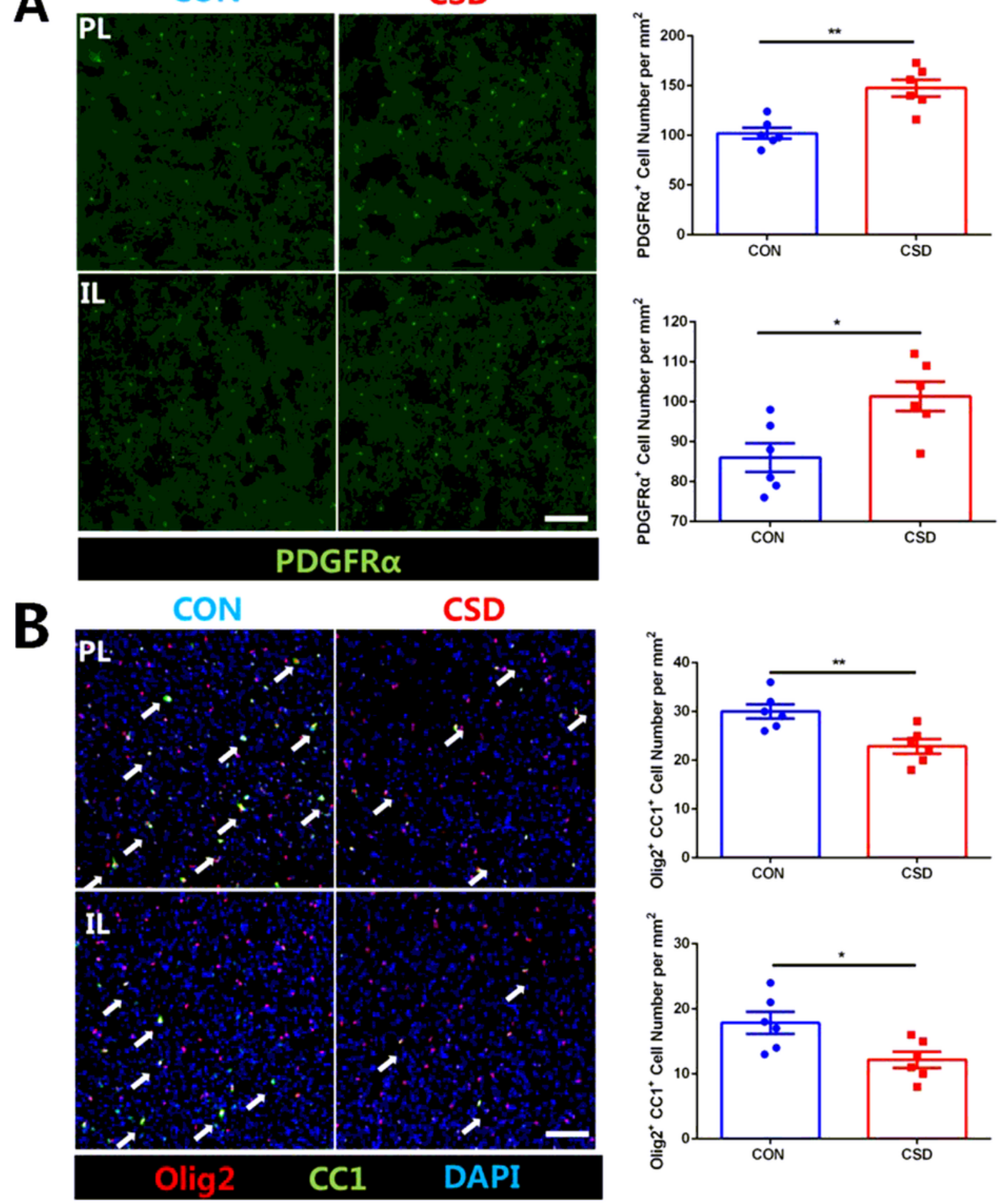


\section{Figure 4}

CSD stress increases OPCs and reduces mature OLs in the mouse mPFC (A) Immunostaining of oligodendrocyte precursor cells (OPCs) (PDGFRa+) and quantification analysis of OPCs in the PL and IL. (B) Immunostaining of mature oligodendrocytes (OLs) (Olig2+ $\mathrm{CC} 1+)$ and quantification analysis of mature OLs in the PL and IL. CON: Control; CSD: Chronic social defeat stress; Scale bar: $100 \mu \mathrm{m}$. Data are shown as mean \pm SEM. ${ }^{*} \mathrm{P}<0.05,{ }^{* *} \mathrm{P}<0.01$. 